\title{
Ratchet behavior in nonlinear Klein-Gordon systems with pointlike inhomogeneities
}

\author{
Luis Morales-Molina* and Franz G. Mertens ${ }^{\dagger}$ \\ Physikalisches Institut, Universität Bayreuth, D-85440 Bayreuth, Germany \\ Angel Sánchez \\ Grupo Interdisciplinar de Sistemas Complejos (GISC) and Departamento de Matemáticas, Universidad Carlos III de Madrid, \\ Avenida de la Universidad 30, 28911 Leganés, Madrid, Spain \\ and Instituto de Biocomputación y Física de Sistemas Complejos, Universidad de Zaragoza, 50009 Zaragoza, Spain
}

(Received 14 October 2004; published 14 July 2005)

\begin{abstract}
We investigate the ratchet dynamics of nonlinear Klein-Gordon kinks in a periodic, asymmetric lattice of pointlike inhomogeneities. We explain the underlying rectification mechanism within a collective coordinate framework, which shows that such a system behaves as a rocking ratchet for point particles. Careful attention is given to the kink width dynamics and its role in the transport. We also analyze the robustness of our kink rocking ratchet in the presence of noise. We show that the noise activates unidirectional motion in a parameter range where such motion is not observed in the noiseless case. This is subsequently corroborated by the collective variable theory. An explanation for this phenomenon is given.
\end{abstract}

DOI: 10.1103/PhysRevE.72.016612

PACS number(s): 05.45.Yv, 05.60.-k, 63.20.Pw, 05.40.-a

\section{INTRODUCTION}

The study of transport mechanisms at the mesoscale level is of great importance nowadays. Specifically, the so-called ratchet systems have been shown to be proper candidates for explaining unidirectional motion in biological systems [1], and have important physical applications for nano- and microscale technologies [2-4]. Many of these models have been developed in the simple picture of pointlike particles [5-7] (see the reviews [8,9] for details). This scenario has been subsequently generalized to spatially extended systems [10-12], where much attention has been paid to situations where the net motion arises through time-symmetry breaking $[13,14]$. This kind of ratchet phenomenon has been recently observed in long Josephson junction (LJJ) devices [15]. Another possibility that has been considered in the literature is that of a nonlinear Klein-Gordon system where the on-site potential is ratchetlike [16]. Notwithstanding, to our knowledge the case of spatial-symmetry breaking by inhomogeneities has not been studied in depth. One such study has been done by Carapella and Costabile who used an inhomogeneous magnetic field to create an effective inhomogeneous junction profile for propagation of fluxons of [17]. Recently an alternative for the generation of motion for extended systems with a disorder in the chain has been proposed [18]. In this procedure the ratchet device is designed from a lattice of pointlike inhomogeneities. For this system, net motion arises from the interplay between disorder and nonlinearity of the nonlinear systems [19].

In this paper we elaborate on the preliminary results reported in [18]. Our aim is to carry out an in-depth analysis of the system, including a careful comparison to related point-

\footnotetext{
*Electronic address: Luis.Morales-Molina@uni-bayreuth.de

${ }^{\dagger}$ Electronic address: Franz.Mertens@uni-bayreuth.de

"URL: http://gisc.uc3m.es/ anxo
}

like ratchets [20] and an extension of our results, originally obtained for the sine-Gordon (SG) model, to other nonlinear Klein-Gordon models such as the $\phi^{4}$ equation. Additional motivation for this work arises from research on models of energy propagation along microtubule filaments inside the cells [21]. This application is specially interesting in view of the possible connection with the dynamics of transport in molecular motors in biological systems, with features similar to those of solitons as extended objects. In this context, the present work sheds light on the role played by the length scale competition between the pointlike inhomogeneities (disorder) and the kinks in the transport dynamics. For this purpose, we use the framework of collective coordinates (CCs) in order to gain insight into the cause of the motion and the degrees of freedom that take part in it. Emphasis will be given to the kink width oscillations and their role in the transport properties. Indeed, in general, the width of the nonlinear topological excitations is crucial for the movement of these coherent excitations. The coupling between the translational and kink width degrees is such that motion takes place [14]. In particular, in the present work we will see that the oscillation range of the kink width is determined by the interplay with the inhomogeneities. An additional, relevant issue is the analysis of the motion dynamics under thermal fluctuations. In ratchets, the noise is an important source of energy and, for some biological systems, it is regarded as the main cause of transport. Here we will consider the robustness of our rocking ratchet system under thermal fluctuations. In this case, activation of unidirectional motion was observed for a certain range of frequencies in the simulations as well as in the CCs.

In order to achieve the above mentioned goals, our paper is organized as follows. In Sec. II we formulate the basis for the ratchet device and explain the origin and physical reasons for the rectification process. The discussion in the $\mathrm{CC}$ framework is devoted to the length scale competition between the inhomogeneities and the kink width, and its influence on the motion dynamics. In the same context, we establish an anal- 
ogy with a simple model used for describing unidirectional motion in molecular motors [20], pointing out the relevant role of the kink width for the ratchet dynamics and its application in biological systems. Subsequently, an analysis of the efficiency in terms of the quantization of the transport is done for the relevant parameters of our ratchet system, including the interference effects among the inhomogeneities. An example is given for the $\phi^{4}$ model, not only for its known rich internal dynamics reinforced by the presence of an internal mode, but also for its potential application to macromolecules (e.g., in transfer of energy in microtubules [21]). Next, in Sec. III, we analyze the kink dynamics subject to noise. In particular, the phenomenon of activation of motion induced by noise is described. In order to explain this behavior, different collective coordinate approaches are implemented and thoroughly discussed. Finally, in the last section we summarize the main contributions of our work and make a discussion about the perspectives on this line. We include appendixes where we detail the $\mathrm{CC}$ approaches for one and two collective variables, extending the generalized traveling wave ansatz (GTWA) to the case where inhomogeneities and noise act together with damping and ac forces.

\section{RATCHET MODEL AND TRANSPORT}

\section{A. Model}

Kink dynamics in the presence of inhomogeneities can exhibit different and interesting behaviors, depending on the interplay between those inhomogeneities and the nonlinearity $[22,23]$ among other factors. The generation of net motion using a lattice of pointlike inhomogeneities is a good example of such nontrivial phenomena [18]. Although in that previous work the problem was discussed for the SG model, it can be generalized in principle for any nonlinear KleinGordon system. Therefore, aiming at that general viewpoint, for our analysis we formulate the model in a general way as follows:

$$
\phi_{t t}+\beta \phi_{t}-\phi_{x x}+\frac{\partial \widetilde{U}}{\partial \phi}[1+V(x)]=A \sin \left(\omega t+\delta_{0}\right)
$$

where $\tilde{U}(\phi)$ is the potential for the nonlinear Klein-Gordon equations and $A \sin \left(\omega t+\delta_{0}\right) \equiv f(t)$ is an external ac force whose parameters $A, \omega$, and $\delta_{0}$ represent the amplitude, frequency, and phase of the periodic force, respectively. The parameter $\beta$ is the damping coefficient. In particular, we will focus on the $\phi^{4}$ and SG models as specific examples; the corresponding nonlinear potentials are $\tilde{U}(\phi)=\frac{1}{4}\left(\phi^{2}-1\right)^{2}$ and $\tilde{U}(\phi)=[1-\cos (\phi)]$, respectively.

For our potential to exhibit a ratchetlike phenomenon, we choose $V(x)$ to be given by a periodically repeated unit cell, formed by an asymmetric array of $\delta$ functions (inhomogeneities). The unit cell configuration, of length $L$ is defined by three inhomogeneities with the same intensity, the first one located at the beginning of the cell, the second at distance $a$ from the first, and the third at distance $b$ from the second. The corresponding mathematical expression is

$$
V(x)=\epsilon \sum_{n}\left[\delta\left(x-x_{1}-n L\right)+\delta\left(x-x_{2}-n L\right)+\delta\left(x-x_{3}-n L\right)\right]
$$

where the parameters satisfy the following constraints: $a, b, c \sim l_{0}$ (static kink width in the absence of inhomogeneities); $a, b<c$ with $a \neq b$, where $L=a+b+c, a=x_{2}-x_{1}, b$ $=x_{3}-x_{2}$, and $c=L+x_{1}-x_{3}$, with $x_{1}<x_{2}<x_{3}$. For our study we have taken $\epsilon>0$, where in the case of the SG model, specifically for LJJs, the pointlike inhomogeneities represent microshorts $[24,25]$. However, the same scheme of arrays of pointlike inhomogeneities can be implemented for $\epsilon<0$, where particularly for the SG model much work has been done [26]. The choice of three inhomogeneities is inspired by biological polymers like DNA where the existence of three bases per codon seems to be the ideal configuration for the occurrence of net transport [27]. In principle it is possible to obtain unidirectional motion by using an array whose configuration presents more than three inhomogeneities per unit cell if the distances between the $\delta$ functions are in the same length scale as the kink width (otherwise, different behaviors could arise, as obtained, e.g., in [23]). However, the inclusion of more inhomogeneities diminishes the efficiency of the transport as we will see below.

\section{B. Simulations of the model}

Contrary to the case of point particles, where motion through pointlike inhomogeneities ( $\delta$ functions) is physically meaningless, in our case we deal with kinks (extended objects) with a determined width. This is an intrinsic feature of these nonlinear excitations, and correspondingly the competition between their width and the distances among the inhomogeneities is crucial for the kink motion. The interference effects among the inhomogeneities [28] create an effective potential for the motion of the kink center, the location of the inhomogeneities determining the direction of motion. For the particular configuration of three inhomogeneities per unit cell, directional motion takes place only under the condition $a \neq b$. In the top panel of Fig. 1, results of simulations of Eq. (1) for the SG case with different values of $a$ and $b$ are depicted. Such a picture shows that our ratchet device is a generic rectifier. In addition, as in standard ratchet systems, the directional motion of the kink center takes place only for certain values of the amplitude of the ac force (see bottom panel of Fig. 1), a behavior that is dependent on the ac force frequency. A more detailed picture of the dynamics of the mean velocity for the SG kink center as a function of the ac force amplitude for different frequencies can be found in [18].

We have restricted ourselves to the overdamped case by taking $\beta=1$, where the inertial effects are small, reducing the generation and propagation of phonons, and for which the kink center moves on a tilted effective potential due to the external ac force. This regime prevents also the dependence on the initial conditions for the dynamics [29]. For the integration of Eq. (1) we have used a Strauss-Vázquez numerical scheme [30] with free boundary conditions and spatial and temporal steps $\Delta x=0.1$ and $\Delta t=0.01$, respectively. We have 

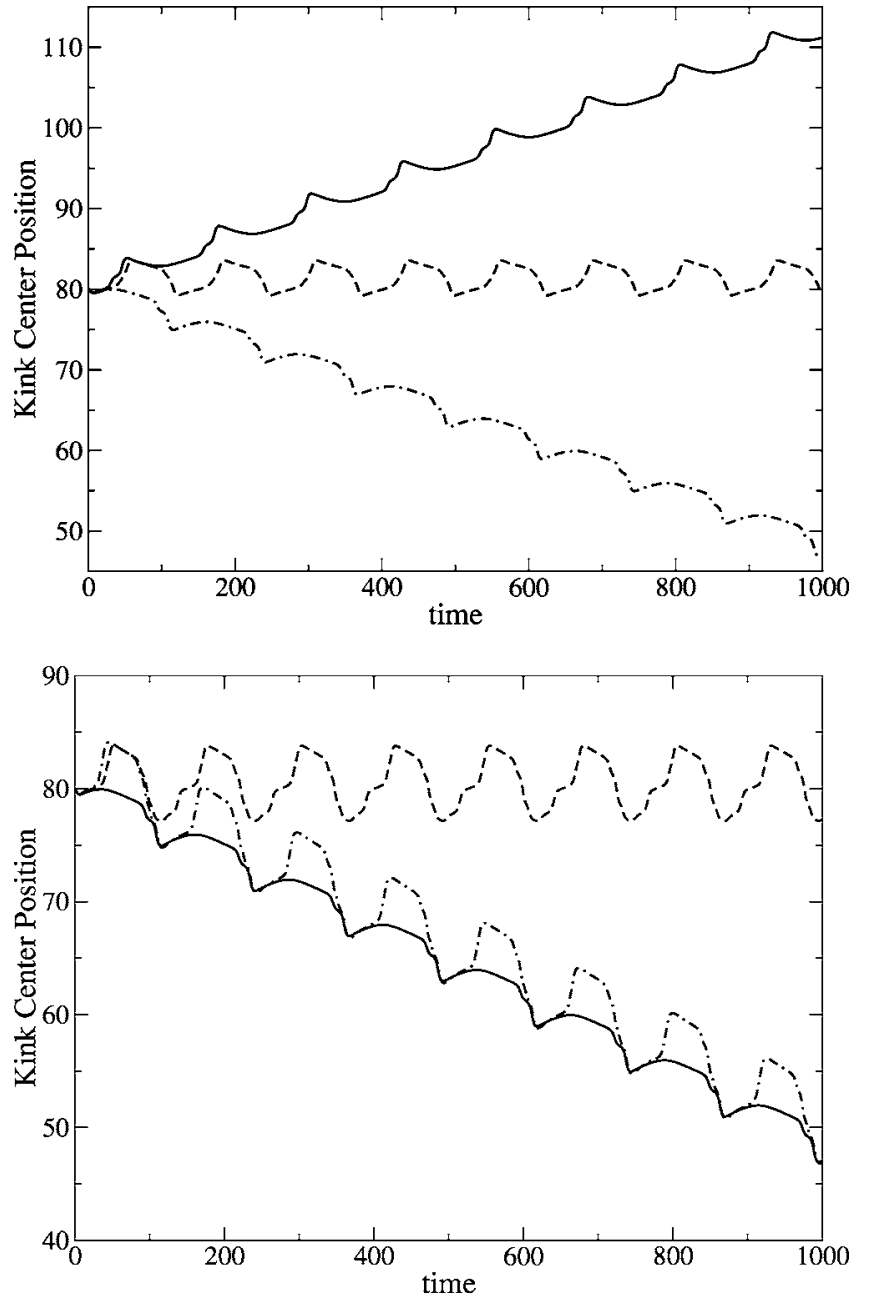

FIG. 1. Simulations of Eq. (1) for SG case; position of kink center vs time. Top panel: Different arrays with the same amplitude of the force $A=0.35: x_{1}=0.5, x_{2}=1.8, x_{3}=2.3(a>b)$ (solid line); $x_{1}=0.5, x_{2}=1.4, x_{3}=2.3(a=b)$ (dashed line); $x_{1}=0.5, x_{2}=1, x_{3}$ $=2.3(a<b)$ (dash-dotted line). Bottom panel: For different amplitudes of the ac force $A=0.35$ (solid line), 0.45 (dashed line), 0.50 (dash-dotted line) with the array $x_{1}=0.5, x_{2}=1, x_{3}=2.3$. The other parameters used are $\beta=1, \omega=0.05, \epsilon=0.8, \delta_{0}=\pi$, and period $L=4$.

checked our results with different spatial steps $\Delta x=0.05$ and 0.02 . The simulations were done for the spatial interval $[-30,150]$ with inhomogeneities arranged periodically according to our unit cell in $[0,120]$.

We have used the following step representation for the $\delta$ function:

$$
\delta\left(x-x_{c}\right) \rightarrow\left\{\begin{array}{cl}
1 / \Delta x, & \left|x-x_{c}\right|<\Delta x / 2, \\
0 & \text { otherwise. }
\end{array}\right.
$$

This representation is not unique but is presumably the simplest form to implement in numerics (see, e.g., [22,28]).

\section{Collective coordinate approach}

In order to understand the dynamics of the kink center different CC approaches have been implemented [18]. In this previous work, it was shown that a 1CC approach is not enough for the correct quantitative description of the results of the simulations even considering possible relativistic effects for high values of ac the force. However, this approach allows us to understand the underlying physics of this ratchet system in the simplest way. On the other hand, satisfactory agreement with the simulations was found by an improved, 2CC approach, which takes into account the kink width dynamics [18]. At this point we will proceed directly with the discussion of the improved theory. The corresponding collective variable equations of two degrees of freedom for Eq. (1) [see Appendix B, Eqs. (B21) and (B22) for D=0] can be expressed as

$$
M_{0} l_{0} \frac{\ddot{X}}{l}+\beta M_{0} l_{0} \frac{\dot{X}}{l}-M_{0} l_{0} \frac{\dot{X} \dot{l}}{l^{2}}=-\frac{\partial U}{\partial X}-q f(t),
$$

$$
\alpha M_{0} l_{0} \frac{\ddot{l}}{l}+\beta \alpha M_{0} l_{0} \frac{\dot{l}}{l}+\frac{1}{2} M_{0} l_{0} \frac{\dot{X}^{2}}{l^{2}}-\frac{1}{2} \alpha M_{0} l_{0} \frac{\dot{l}^{2}}{l^{2}}=-\frac{\partial U^{\text {int }}}{\partial l}-\frac{\partial U}{\partial l},
$$

where $X$ stands for the position of the kink center, $l$ represents the kink width, the internal potential energy of the kink is

$$
U^{\mathrm{int}}=\frac{1}{2} M_{0}\left(\frac{l_{0}}{l}+\frac{l}{l_{0}}\right)
$$

and $U(X, l)$ is an effective potential depending on the specific equation one is dealing with. In particular, for the SG case $M_{0}=8, l_{0}=1, \alpha=\pi^{2} / 12, q=2 \pi$, and the effective potential is given by

$$
\begin{aligned}
U(X, l)= & 2 \epsilon \sum_{n}\left[\frac{1}{\cosh ^{2}\left[\left(X-x_{1}-n L\right) / l\right]}\right. \\
& \left.+\frac{1}{\cosh ^{2}\left[\left(X-x_{2}-n L\right) / l\right]}+\frac{1}{\cosh ^{2}\left[\left(X-x_{3}-n L\right) / l\right]}\right] .
\end{aligned}
$$

As in the case of simulations we restrict ourselves to the overdamped case (taking $\beta=1$ ).

As we can see from the previous equations the kink width dynamics is coupled to the motion of the center of the kink. Therefore, changes in the kink width directly affect the translational motion. It is possible to observe, for instance, that decreasing the kink width decreases the effective ac force, making it necessary to increase the amplitude of the ac force in order to compensate for this effect. This is an important factor that explains in part the shift observed in the locations of the windows of motion of the simulations with respect to those obtained from the 1CC approach [see Fig. 1(b) in [18]]. Another relevant conclusion is the feedback between the effective potential landscape and the kink width, determined in turn by the potential. In this fashion, the $2 \mathrm{CC}$ approach reflects the nontrivial interaction of the kink with the inhomogeneities, which is otherwise known to exhibit many counterintuitive phenomena [28].

To deepen our understanding of the dynamics, let us look into the oscillations of the kink width. 


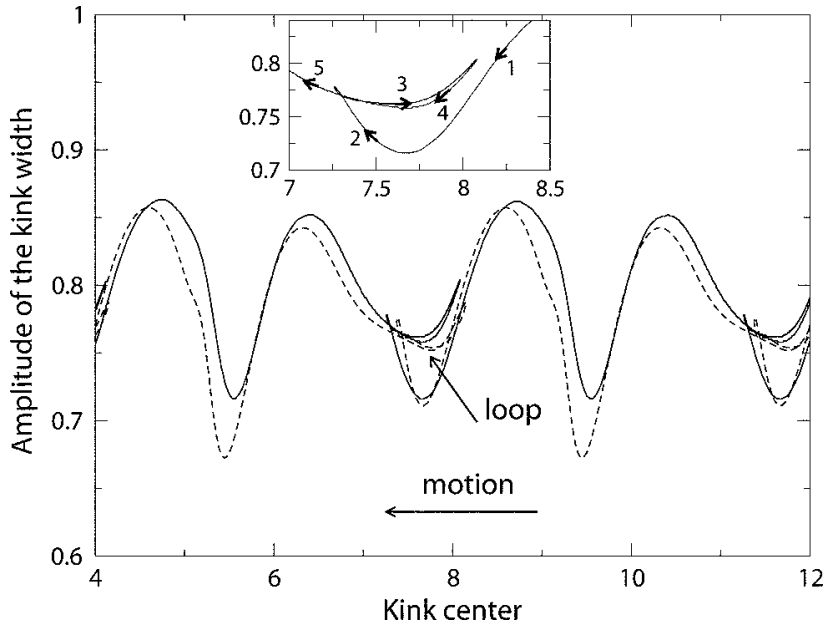

FIG. 2. SG case: Amplitude of the kink width versus kink center. Simulation (solid line); 2CC approach Eqs. (4) and (5) (dashed line). The parameters are $\omega=0.1, A=0.44$ for the array $x_{1}=0.5, x_{2}$ $=1, x_{3}=2.3$, period $L=4$, and $\epsilon=0.8$. See text for a discussion of the loop. Inset: enlargement of the loop indicated by an arrow in the main figure. The motion of the kink center is indicated by numbered arrows.
A picture of the kink width oscillations versus kink center position is shown in Fig. 2. As we can see, the agreement with the CC approach is excellent, confirming the validity of our predictions. The existence of loops is particularly interesting; they arise as a consequence of the kink center motion back and forth into the wells of the effective potential (see Fig. 3), i.e., describing half an oscillation before overcoming the barrier.

Interestingly, another feature that stands out clearly is that the oscillations are around a value different from $l_{0}=1$, the width of the unperturbed kink. Figure 2 shows that they take place around $\bar{l} \approx 0.8$ and, furthermore, that $l_{0}$ is not even included in the range of oscillations. This phenomenon is the result of the balance between two opposite effects. (a) On one hand, the inclusion of inhomogeneities increases the potential energy of the system. This fact is reflected in the effective potential energy landscape Fig. 3. This picture shows that when the kink width decreases, the potential energy decreases as well. Taking two points with the same value for $X$ but with different kink widths $l$, for example $M$ and $O$ in the bottom panel of Fig. 3, we see this difference in potential energy, i.e., $U_{M}>U_{O}$ where $l_{M}>l_{O}$. Therefore, as the tendency of the system is to go to the minimum of the potential energy, the kink width will decrease. (b) On the other hand, the kink internal potential energy Eq. (6) has a minimum at $l_{0}$, and hence the energy increases when the kink width decreases (see Fig. 4 for $l<l_{0}$ ); notice that the first term of this equation accounts for a repulsive interaction and
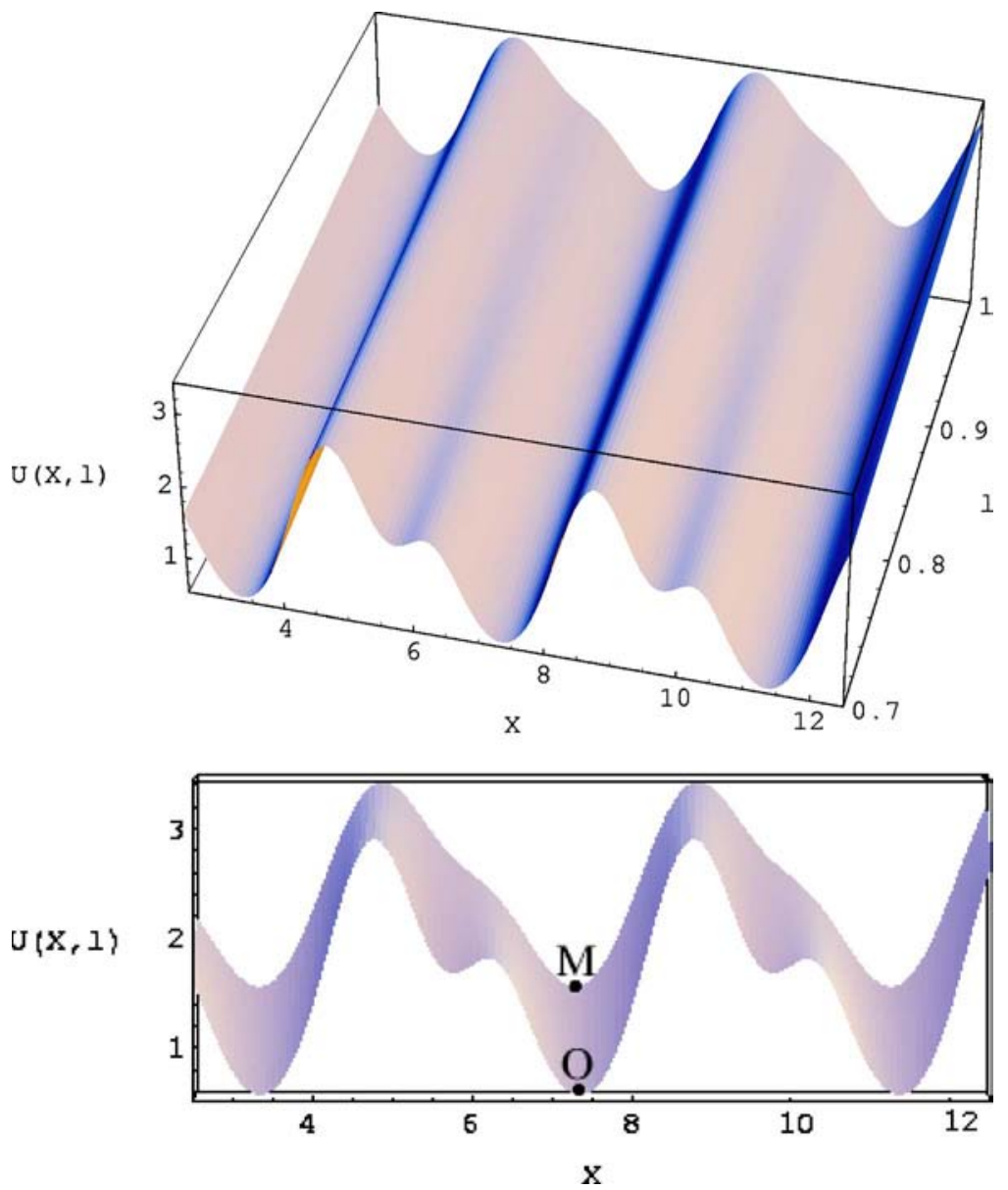

FIG. 3. (Color online) Top panel: 3D plot of the effective potential Eq. (7). Bottom panel: Frontal view rotated by a small angle. The array used is the same as in Fig. 2. 


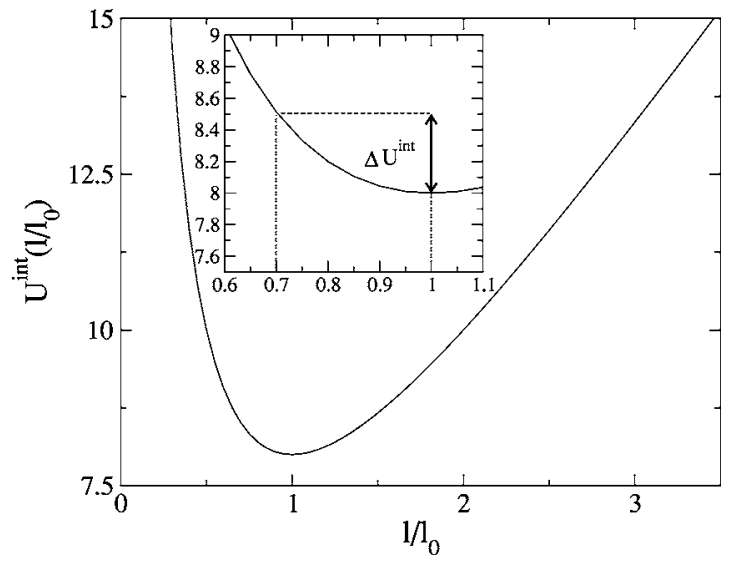

FIG. 4. Internal potential energy vs normalized kink width, Eq. (6) (SG case). The inset shows the part of curve where the oscillations of the kink width take place.

the second is given by an attractive one. As a result of the balance between (a) and (b), a new minimum will appear for the oscillations of the kink width. It is important to note that the difference $\Delta U^{\text {int }}$ of the internal potential energy for the kink width $l=0.7$ with respect to the value $l_{0}=1$ (inset of Fig. 4 ) is of the same order as the energy difference $U_{M}-U_{O}$ between the points mentioned before for the effective potential introduced by the inhomogeneities, in agreement with this discussion.

\section{Related point particle models}

A problem closely related to our $2 \mathrm{CC}$ approach, given by a point particle ratchet with two degrees of freedom, has been studied in [20]. This model was designed for describing molecular motor dynamics consisting of two particles joined by a spring moving in a ratchet potential. The corresponding equations of motion are given by

$$
\begin{aligned}
& \dot{u}_{1}=-\frac{\partial V\left(u_{1}\right)}{\partial u_{1}}-\frac{\partial W\left(u_{2}-u_{1}\right)}{\partial u_{1}}+A \sin (\omega t)+\xi_{1}(t), \\
& \dot{u}_{2}=-\frac{\partial V\left(u_{2}\right)}{\partial u_{2}}-\frac{\partial W\left(u_{2}-u_{1}\right)}{\partial u_{2}}+A \sin (\omega t)+\xi_{2}(t),
\end{aligned}
$$

where $u_{1}, u_{2}$ represent the coordinates of the particles, $V$ is a sawtooth potential, and $W$ is the internal potential energy. Here $\xi_{i}$ with $i=1,2$ is Gaussian white noise. Ignoring the noise terms and their influence on the net motion, we see that the change of variables $X=\frac{1}{2}\left(u_{1}+u_{2}\right)$ and $l=u_{2}-u_{1}$ casts the system in a similar shape as Eqs. (4) and (5) in the overdamped case, for which in good approximation the inertial terms could be neglected. In such a context the variables $X, l$ can be interpreted as the mass center and elongation (distance between the particles), respectively, and obviously they resemble the variables mass center and width of the kink in our system.

Notice that in both models we have an asymmetric potential. In our case it is given by Eq. (7), which is asymmetric at the CC level if the already mentioned conditions for the distances between the inhomogeneities are satisfied. In both systems, there are internal potential energies that characterize their elastic properties. In the model in [20], the internal potential is expressed through a harmonic function (in the original variables)

$$
W\left(u_{1}, u_{2}\right)=\frac{1}{2} k\left[\left(u_{2}-u_{1}\right)-l_{0}\right]^{2},
$$

which in our collective coordinates can be written as

$$
W(l)=\frac{1}{2} k\left[l(t)-l_{0}\right]^{2},
$$

where $k$ is the elasticity constant.

The links between the two models can be made more explicit by using a value for $l_{0}$ close to the minimum around which the kink width oscillates in our simulations (cf. the discussion in Sec. II C).

It is important to point out that we have defined $l$ as a kink width variable as given in the expression (B7) of Appendix B. However, what the quantity $l$ actually means is the distance at which the kink shape approaches its asymptotic value, measured from the center. This means that $l$ in our notation is half the "real kink width." Consequently the ratio (real kink width)/(period of the effective potential) becomes $2 \bar{l} / L \approx 0.4$ for which a very interesting dynamics for point particle dynamics has been reported in the related twoparticle model [31].

This comparison between our model and that in [20] allows us to point out their main differences as well. It is particularly important that in our framework, the internal energy can describe satisfactorily the repulsive interaction between real molecules where a van der Waals-like force prevents their overlap. This is very close to what occurs in molecular motors: if we take again the motion of kinesin as an example, this molecule has two dimer heads that act as "feet," allowing the molecule to "walk" along a microtubule [32]. The repulsion will then appear when the two dimer heads are too close. Such a repulsive interaction cannot be naturally accounted for within the model of two particles. For solving this problem the authors of [20] resort to fixing arbitrary values for $l_{0}$ which in our case is not necessary. Note, however, that in spite of the technical differences between the two models, phenomenologically they are very similar: both of them try to understand how the motion of molecular motors, which proceeds in steps accompanied by deformations (in the case of kinesin, when one step advances in front of the other), can arise. The common conclusion is that a point particle ratchet would not be a good model because a second degree of freedom is needed to capture the whole mechanism of the motion. The advantage in our approach is that this second degree of freedom arises on its own, without a priori constructions, as an emergent property of the nonlinear excitation. Recent studies [31,33] show similar phenomena for the two degrees of freedom point particle ratchet of [20] when the ratchet is of flashing type. The close relationship of the model of [20] to ours suggests that nonlinear Klein-Gordon models can also exhibit rectification working as flashing ratchets, an issue we will address in future work. 


\section{E. Length scales and quantization of transport}

It should be clear from the results discussed so far that in order to obtain a ratchet device for extended nonlinear systems with topological nonlinear excitations, the configuration of the inhomogeneities should be designed in such a way that the distance between the inhomogeneities is of the order of the kink width. However, this picture is somewhat too simple, and as we will see below, another important factor to take into account is the existence of interference effects. Naively, one may try to design a similar ratchet system for the $\phi^{4}$ equation. Considering only the kink width factor, it would seem that on enlarging the SG array by a factor of $\sqrt{2}$ (the ratio between the kink widths in the two models) similar phenomena would be observed.

Let us make a more specific comparison between both models. To this end, we use the $1 \mathrm{CC}$ framework in the nonrelativistic approach, where the equation of motion can be written as

$$
\ddot{X}+\beta \dot{X}=-\frac{d u}{d X}-\frac{q A}{M_{0}} \sin \left(\omega t+\delta_{0}\right),
$$

where $u=U / M_{0}$ is the normalized effective potential. For the SG case we have the following expression:

$$
u(X)=\frac{2 \epsilon}{M_{0}} \sum_{n} \sum_{i=1}^{3} \frac{1}{\cosh ^{2}\left[\left(X-x_{i}-n L\right) / l_{0}\right]}
$$

with $l_{0}=1$ and $M_{0}=8$, whereas for the case of $\phi^{4}$ we have

$$
u(X)=\frac{\epsilon}{4 M_{0}} \sum_{n} \sum_{i=1}^{3} \frac{1}{\cosh ^{4}\left[\left(X-x_{i}-n L\right) / l_{0}\right]}
$$

with $l_{0}=\sqrt{2}$ and $M_{0}=2 \sqrt{2} / 3$. The parameter $q$ is the topological charge and it is given by $q=2 \pi$ and $q=2$ for the SG and $\phi^{4}$ systems, respectively. The normalized effective potentials for two different arrays are depicted in Fig. 5. Figure 5(a) shows standard asymmetric potentials for ratchet systems obtained with an array that satisfies the conditions mentioned above for the location of the inhomogeneities in the SG case. However, in Fig. 5(b) the effective potentials obtained for an array approximately given by the multiplication by the factor $\sqrt{2}$ of the first one, shows a local minimum similar to an array of asymmetric double-well traps. This last one has been used as a device for generating motion of vortices in superconductor materials [34].

According to the our previous arguments based on the kink width role, a similar picture is expected for the normalized effective potential of the $\phi^{4}$ and SG systems if the arrays satisfy the same length ratio as the full systems. Strikingly, Fig. 5 shows that the normalized effective potentials are almost the same but for the same array length. This apparent discrepancy can be explained if we take a detailed look at the potential given by Eqs. (13) and (14) for both cases $\left(\mathrm{SG}\right.$ and $\left.\phi^{4}\right)$. It is clear from those expressions that, while in the case of the $\phi^{4}$ model we have a $\cosh ^{4}$ factor in the denominator, the SG model has a $\cosh ^{2}$ factor. Therefore, the peaks and valleys in the effective potential for the $\phi^{4}$ system are much narrower than for the SG system, thus compensating for the increment in length. In addition, in the SG
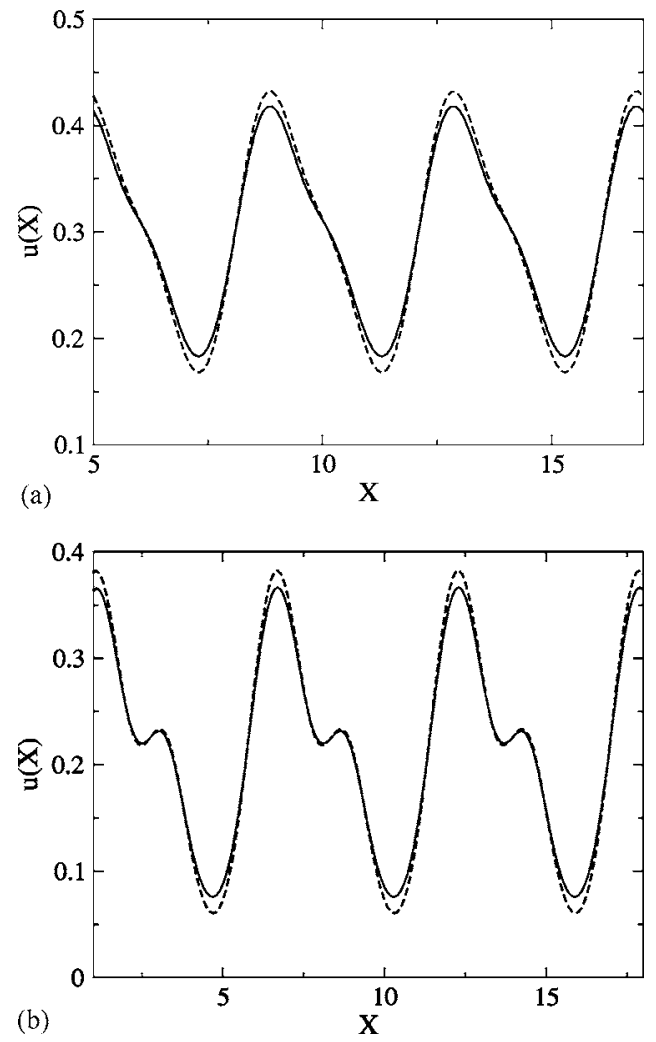

FIG. 5. Normalized effective potential for the kink center within the CC approach, Eqs. (13) and (14), for two different $\delta$ peak arrays with $\epsilon=0.8$. (a) $x_{1}=0.5, x_{2}=1, x_{3}=2.3, L=4$. (b) $x_{1}=0.7, x_{2}=1.4$, $x_{3}=3.2, L=5$.6. In both panels SG model (solid line) and $\phi^{4}$ model (dashed line).

model we will have dynamical changes of the effective potential due to the kink width variations, making more complicated the dynamics of motion. In any event, these effective potentials obtained in the simple approach highlight the importance of interference effects (see also [23,28]) and make it clear that the kink width is not the unique quantity to take into account.

The consequences of choosing either the first or the second array for the kink dynamics are revealed in Fig. 6. We have taken for the analysis the $\phi^{4}$ model with a relative low frequency for the ac force, for which the mean velocity as a function of the ac force amplitude shows a staircase structure. The range for the amplitude values was taken from the following rescaling expression: $q^{\phi^{4}} A^{\phi^{4}} / M_{0}^{\phi^{4}}=q^{\mathrm{SG}} A^{\mathrm{SG}} / M_{0}^{\mathrm{SG}}$. This relation is deduced from the comparison between the $1 \mathrm{CC}$ approaches for $\mathrm{SG}$ and $\phi^{4}$ models, considering the similarity of the normalized potentials discussed above. Figure 6 shows the dependence of the kink mean velocity as a function of the amplitude for two different arrays and heights of the perturbations introduced by the inhomogeneities. As we can see, the motion is quantized as in standard ratchet systems $[5,7]$ and is characterized by the existence of gaps for which the net motion is absent (i.e., pure oscillating states). The absolute value of the mean velocity can be expressed as $|\langle d X / d t\rangle| \equiv|\langle V\rangle|=(L \omega / 2 \pi)(m / n)$ as usual [35], where the indices $m, n \in \mathbb{N}$ quantize the motion. Using the expression for $|\langle V\rangle|$ we can characterize the motion for each frequency and 


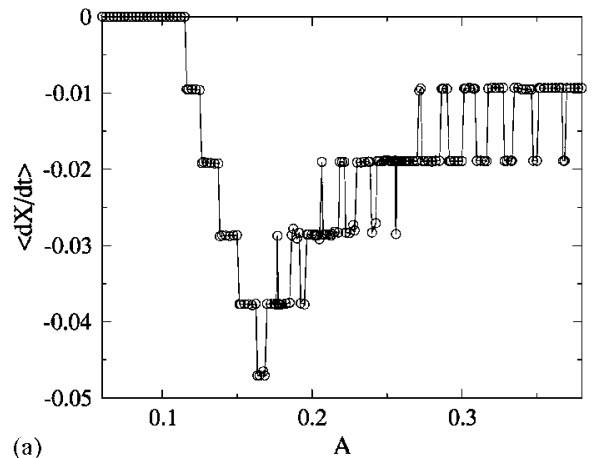

(a)

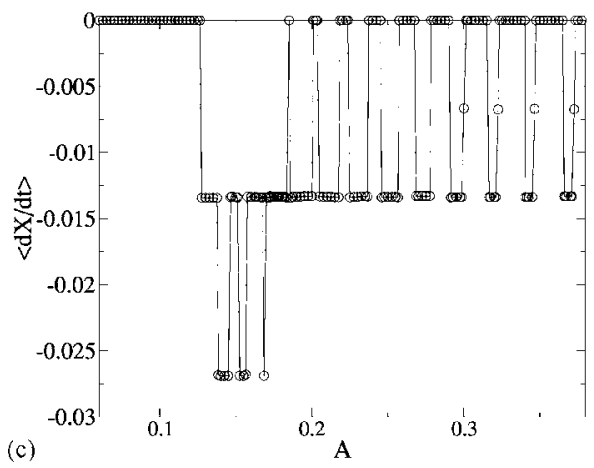

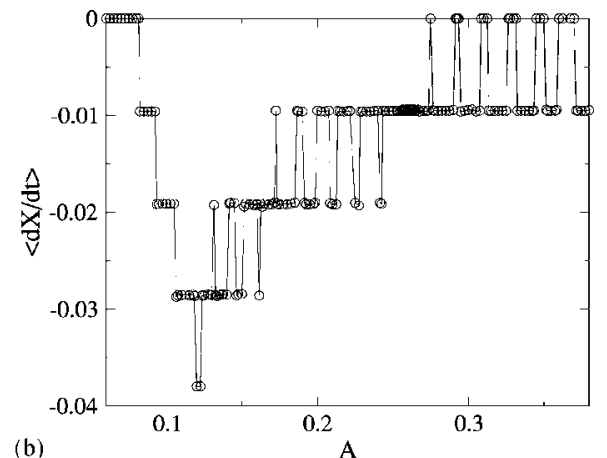

(b)

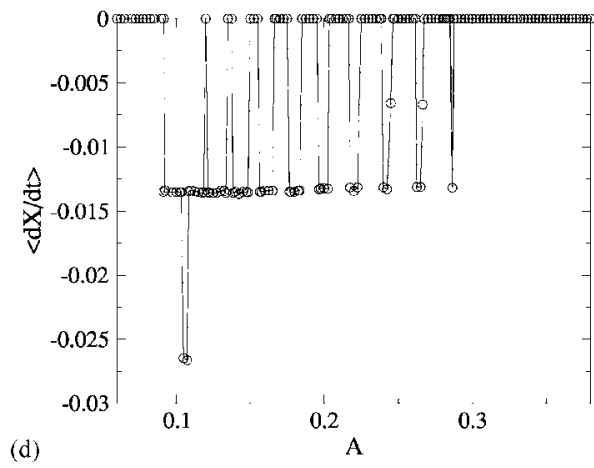

FIG. 6. Simulation results for $\phi^{4}$. Mean velocity vs driving amplitude $A$ for the frequency $\omega$ $=0.015$. (a) $\epsilon=0.8, x_{1}=0.5, x_{2}=1$, $x_{3}=2.3, L=4$. (b) $\epsilon=0.6, x_{1}=0.5$, $x_{2}=1, x_{3}=2.3, L=4$. (c) $\epsilon=0.8$, $x_{1}=0.7, x_{2}=1.4, x_{3}=3.2, L=5.6$. (d) $\epsilon=0.6, \quad x_{1}=0.7, \quad x_{2}=1.4, \quad x_{3}$ $=3.2, L=5.6$. The lines connecting the points serve as guides for the eye. period of the array. Comparing the values obtained from the simulations with the results derived from the expression for $|\langle V\rangle|$ with corresponding parameters $L$ and $\omega$, we find that $m$ and $n$ can take the following values. For Fig. 6(a), $m$ $=1,2,3,4,5$ and $n=1$; for Fig. 6(b), $m=1,2,3,4$ and $n=1$; for Fig. 6(c), $m=1,2$ with $n=1$ and 2; and for Fig. 6(d), $m$ $=1,2$ with $n=1$ and 2 . Although the mean velocity modulus increases with the period $L$, the maximum value of the index $m$ significantly decreases, leading to a global decreasing of the velocity. These results prove that the inclusion of more inhomogeneities per unit cell, which obviously enhances the period $L$, is not a good option if we want to reach high velocities. Furthermore, a very low frequency would be required to obtain windows of motion. In the case of the dependence on the inhomogeneities height, the starting point of the stair-step structure shows a shift toward greater amplitude of the ac force when the height is increased, which is natural in order to overcome the barrier. Nevertheless, a higher speed is found, arising from a higher $m$ and observable also as a broadening in the windows of motion.

\section{DYNAMICS UNDER NOISE}

This far, we have analyzed the ratchetlike behavior of our system in the deterministic case. However, it is clear that for our model to be more realistic, for instance, in the context of LJJs, the effect of the temperature has to be taken into account. The behavior of ratchet systems for nonzero temperature has been extensively studied both for point particles $[5,6,36-38]$ and for nonlinear extended systems [10,12,39]. As for the problem we are considering here, the fact that there has not been much effort on soliton ratchetlike phenomena induced by spatial inhomogeneities carries over to the stochastic effects. Therefore, it is important that we address this issue here. For the present work, we will focus on the robustness of our rocking ratchet under thermal fluctuations. Another relevant issue would be the possible activation, resonance, or modification of the transport features induced by noise, but this topic deserves a detailed analysis and will be the subject of future work.

\section{A. Full model}

For the sake of definiteness, we consider the SG model under the influence of a Gaussian white noise; the results for the $\phi^{4}$ equation are similar. Introducing the effect of the temperature through the fluctuation-dissipation relationship and considering the overdamped case as before, taking $\beta$ $=1$, we find the following equation:

$$
\phi_{t t}+\phi_{t}-\phi_{x x}+\sin (\phi)[1+V(x)]=f(t)+\eta(x, t),
$$

with

$$
\begin{gathered}
\langle\eta(x, t)\rangle=0, \\
\left\langle\eta(x, t) \eta\left(x^{\prime}, t^{\prime}\right)\right\rangle=D \delta\left(x-x^{\prime}\right) \delta\left(t-t^{\prime}\right),
\end{gathered}
$$

where $f(t) \equiv A \sin \left(\omega t+\delta_{0}\right)$ and the intensity of the noise $D$ $=2 k_{B} T$.

For the numerical simulations of the full partial differential equation as well as for the numerical solution of the collective variables (to be discussed in Sec. III B), we have used the Heun method with the Box-Muller-Wiener algorithm for generating Gaussian random numbers of mean zero and variance 1 [40]. In Fig. 7 we show the behavior of the kink center dynamics under thermal fluctuations. Hereafter, we have set the array parameters to be $x_{1}=0.5, x_{2}=1, x_{3}$ 

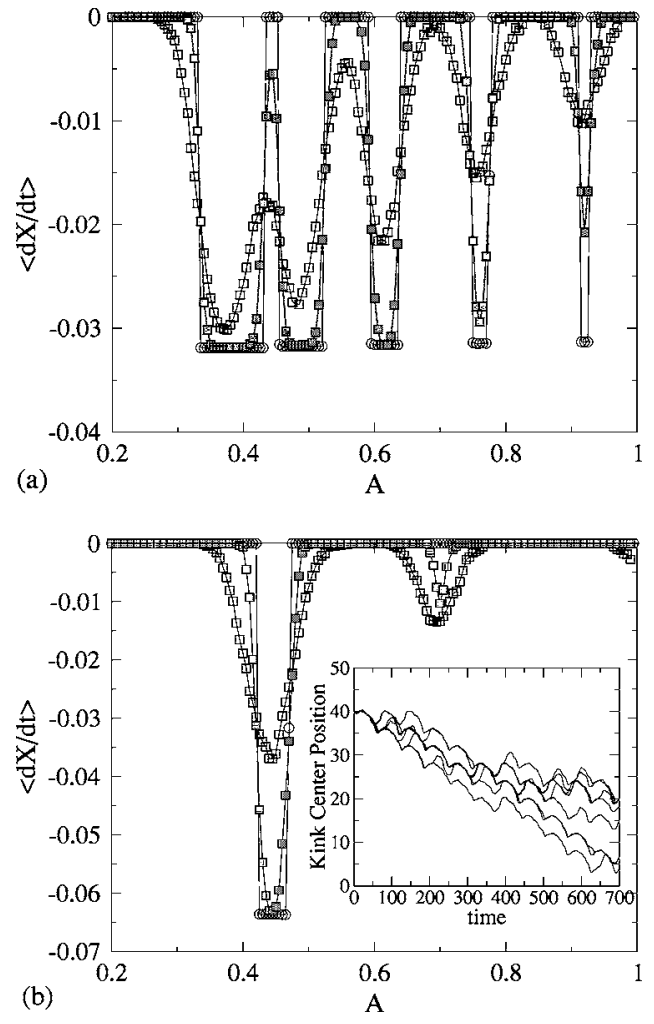

FIG. 7. Mean kink velocity $\langle d X / d t\rangle$ vs driving amplitude $A$ for different intensities of the noise. (a) $\omega=0.05 ; D=$ (circles) 0 , (filled squares) 0.005 , (squares) 0.05 . (b) $\omega=0.1 ; D=$ (circles) 0 , (filled squares) 0.005 , (squares) 0.05 . Lines serve as guides for the eye. The inset shows several realizations for the motion of the kink center with $A=0.43, \delta_{0}=\pi$, and $D=0.05$.

$=2.3, L=4$, and $\epsilon=0.8$ for our study. The mean velocity was calculated using the expression in [8], namely,

$$
\langle V\rangle=\langle\dot{X}\rangle=\lim _{t \rightarrow \infty} \frac{\langle X(t)-X(0)\rangle}{t},
$$

where the average is to be understood over many realizations of the noise. From this figure we see that the steps of the deterministic case are now smoothed, a typical feature for the dynamics under noise. It is important to realize that this smoothing affects the regions between the windows, which become minima of the mean velocity modulus $|\langle V\rangle|$ instead of gaps with null mean velocity [see Fig. 7(a)]. This phenomenon is directly related to the strength of the noise, i.e., when the noise increases, the absolute value for the mean velocity decreases but simultaneously the connection between the windows becomes more evident and the windows of motion become less pronounced.

As in most other ratchet systems, in our model the stochastic fluctuations due to temperature assist the jumps of the kink center from one well to the next one, allowing in some cases jumps in the opposite direction to the rectification [see the inset graphic in Fig. 7(b)] which is not possible in the absence of noise. Accordingly, the thermal fluctuations affect the mechanism of rectification whereas, on the other hand, they yield the dynamics of the pure oscillating states of the

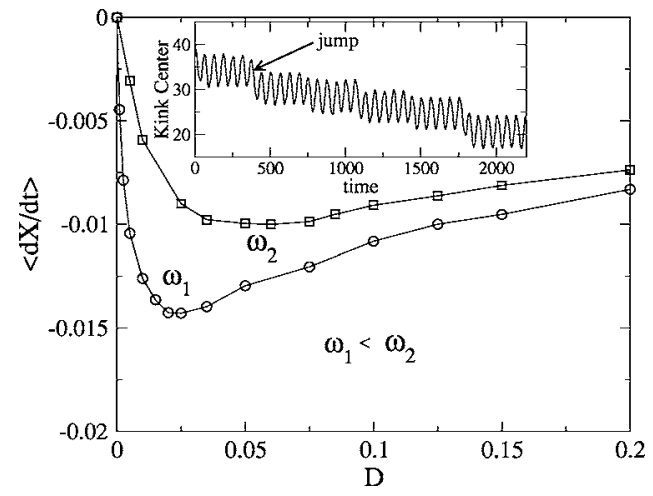

FIG. 8. Mean kink velocity $\langle d X / d t\rangle$ vs intensity of noise $D$. Circles, $\omega=0.1$ and $A=0.70$; squares, $\omega=0.11$ and $A=0.75$. Inset shows one realization for the motion of the kink center for $\omega=0.1$, $A=0.70, \delta_{0}=0$, and $D=0.005$.

kink center unstable (i.e., destabilizing the regions with locked directional motion for zero temperature). The joint action of both effects leads to the smoothing of the windows and the connection of the deterministic gaps. For relatively high temperatures the thermal kink energy is sufficient for overcoming the barrier of the effective potential, and the kink motion is in practice diffusive, the influence of the barrier becoming negligible. For this reason the rectification of motion takes place only for not so large values of the noise intensity (see discussion in [41]).

A remarkable feature we have observed in the simulations is shown in Fig. 7(b) for frequency $\omega=0.1$, where new windows (absent in the deterministic case) appear. This scenario is very similar to the one reported in [12] where a similar surprising and intriguing phenomenon was noted. There, the authors discussed that these new windows arose due to jumps of the fluxons between stable and unstable pinned fixed points of the deterministic dynamics. Considering the interest in this stochastic phenomenon, we carried out a careful analysis of the corresponding zone. To summarize this work, in Fig. 8 we plot the mean velocity as a function of the noise intensity for different values of the frequency, showing the existence of an optimal value for the intensity of the noise for which a maximum absolute value of the mean velocity is obtained. The inset in Fig. 8 makes it clear that, as expected and suggested in [12], the mechanism of activation occurs through jumps between multistable states (states of the kink center which in the absence of noise are purely oscillating). Therefore, a higher velocity is obtained when the residence time in these multistates is reduced or, in other words, when the intervals between consecutive jumps decrease. Once again, this process of activation becomes more effective when the noise intensity increases, but above a certain value of the noise intensity the kink center starts to jump in the direction opposite to that of the rectification, leading to a global loss in efficiency. This explains the existence of an optimal value for the noise intensity for which the velocity reaches a maximum value.

Another interesting characteristic observed in Fig. 8 is the dependence of the maximum mean velocity on the frequency. Specifically, for a frequency value slightly larger than $\omega=0.1$, the maximum velocity decreases, the peak mov- 
ing toward greater values of the noise and the corresponding window of motion moving toward greater values of the ac force. Accordingly, for a relatively large value of the frequency, above $\omega=0.11$, the window of motion induced by noise disappears. On the other hand, for frequencies slightly smaller than $\omega=0.1$, a new window in the absence of noise is obtained. With all these results, it is clearly established that the unidirectional motion induced by noise occurs only for a narrow window of frequency values.

We will show in Sec. III B that this phenomenon seems to be a general feature, since at the $\mathrm{CC}$ level the system behaves very much like the dynamics of point particles.

\section{B. Collective coordinates in presence of noise}

In order to understand the behavior observed in the previous section we resort again to the $\mathrm{CC}$ approach. As a first step, we take into account only one degree of freedom. Although, as discussed above, this framework is inaccurate for describing quantitatively the kink motion on a lattice of inhomogeneities, it does help understand qualitatively most of the features observed in the simulations, without unnecessary analytical complications. After some algebra (see Appendix A for details), with $\beta=1$, we find the following expression for the kink center coordinate with noise:

$$
M_{0} \ddot{X}+M_{0} \dot{X}=-\frac{d U}{d X}-q f(t)+\sqrt{D M_{0}} \xi(t)
$$

with $\langle\xi(t)\rangle=0,\left\langle\xi(t) \xi\left(t^{\prime}\right)\right\rangle=\delta\left(t-t^{\prime}\right)$. For the sake of simplicity we have taken the nonrelativistic approach $\dot{X}^{2} \ll 1$, for which the noise turns out to be simply additive.

Figure 9 presents the results of the numerical integration of Eq. (18). Much as we did in the simulations, we calculate the mean velocity using Eq. (17), taking up to 500 realizations. From this plot two main features also observed in the simulations can be seen. First, smooth curves are obtained for the mean velocity as a function of the amplitude of the ac force, with values that decrease when the noise is increased.

Second, new windows appear, and inside them there is a value of the noise intensity for which the velocity reaches a maximum value (inset in Fig. 9). It is thus evident that, in spite of the quantitative differences with the simulations, this simple approach does predict correctly the qualitative behavior of the full system.

In order to improve the results presented so far, we have extended the framework to two collective variables. By doing so (see Appendix B) we arrive at Eqs. (B21) and (B22) with two uncorrelated multiplicative white noises, meaning that the stochastic driving terms depend on the kink width dynamics.

The results for this improved approach are collected in Fig. 10. Comparing with the simulations (Fig. 7), we can observe the excellent agreement, with the locations of the windows correctly predicted. As expected the curves are again smooth, a feature correctly accounted for already in the $1 \mathrm{CC}$ framework. For the frequency $\omega=0.1$, a new window is predicted, whose location is also in very good agreement with its corresponding window in the simulations. These results confirm the importance of considering the kink width

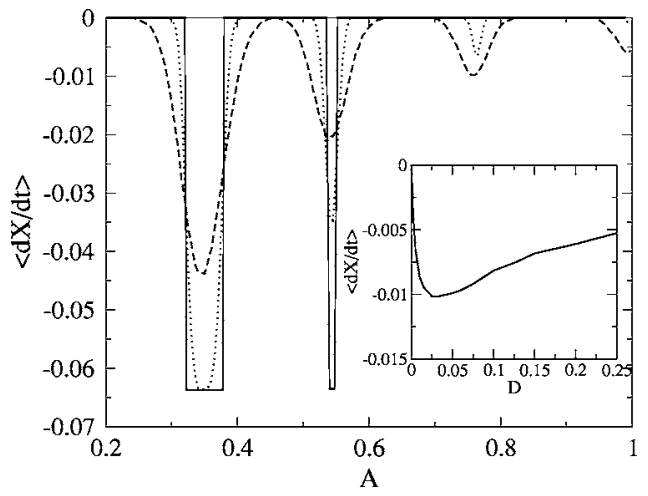

FIG. 9. CC approach: Mean kink velocity $\langle d X / d t\rangle$ vs driving amplitude $A$ for different intensities of the noise and frequency $\omega$ $=0.1$. Solid line, $D=0$; dotted line, $D=0.005$; dashed line, $D$ $=0.05$. Inset: Mean kink velocity $\langle d X / d t\rangle$ vs noise intensity $D$ for $A=0.7625$.

dynamics in the framework of the collective coordinates in order to achieve correct quantitative results as compared to the simulations.

\section{CONCLUSIONS}

We have studied the dynamics of solitons in a lattice of pointlike inhomogeneities in two different nonlinear systems,
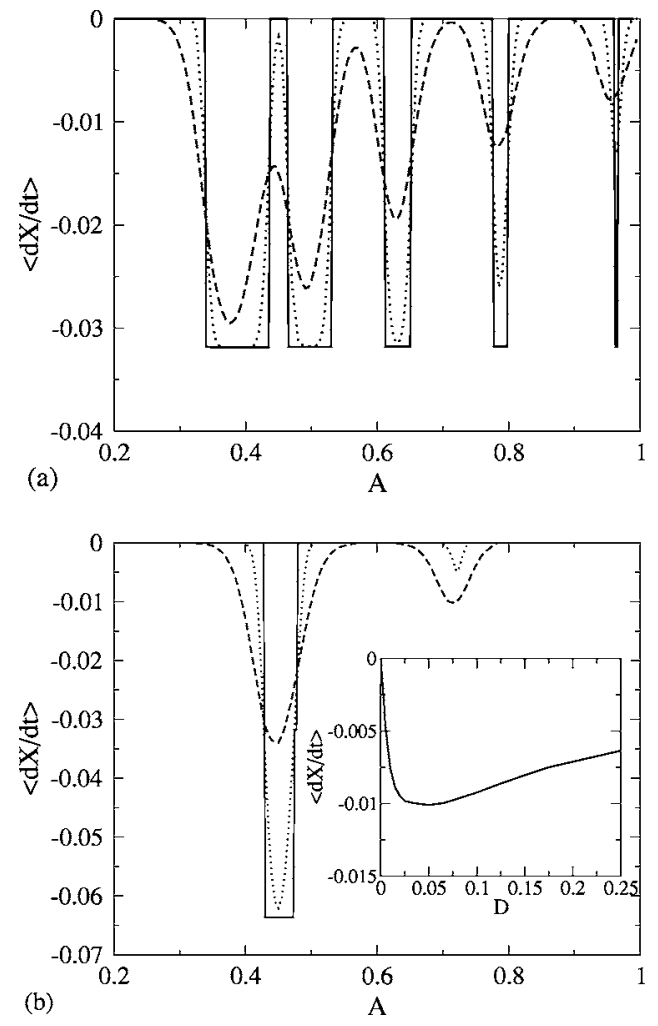

FIG. 10. CC approach for two degrees of freedom [Eqs. (B21) and (B22) of Appendix B]. Mean kink velocity $\langle d X / d t\rangle$ vs driving amplitude $A$ for different intensities of the noise. (a) $\omega=0.05 ; D$ $=($ solid line) 0 , (dotted line) 0.005, (dashed line) 0.05. (b) $\omega=0.1$; $D=$ (solid line) 0 , (dotted line) 0.005 , (dashed line) 0.05. Inset shows mean kink velocity $\langle d X / d t\rangle$ vs noise intensity $D$ for $A$ $=0.72$ with $\omega=0.1$. 
the SG and the $\phi^{4}$ models, as good representatives of an entire class of nonlinear models. We have designed a periodic lattice made of an asymmetric unit cell, where, depending on the lattice parameters, ratchetlike motion of solitons (rectification) is observed. This is one of the few examples proposed so far for soliton ratchets based on spatial inhomogeneity. Building on a preliminary work reported in [18], we have analyzed in full detail all the characteristics and features of interest of our model. In order to understand the observed phenomenology, we have developed two CC approaches: one that takes into account only the motion of the kink center, and another one that includes the kink width as a second degree of freedom. We have seen that whereas both techniques give good qualitative results and allow for a general understanding of the phenomenon, only when the kink width enters in the $\mathrm{CC}$ description are the results quantitatively correct. Interestingly, the CC approach allows us to show that rectification takes place only when the unit cell gives rise to an effective potential of the same length scale as the kink width. This fact, along with the relevant role played by the width dynamical behavior, makes us conclude that one essential ingredient for observing ratchetlike behavior in this system is the existence of length scale competition [19].

As a necessary complement to the deterministic study, we have considered the influence of thermal noise on the behavior of our model systems. As in the deterministic case, the $\mathrm{CC}$ technique yields very good results. Of particular relevance is the appearance, at the $2 \mathrm{CC}$ level, of multiplicative noise in the CC equations, coupled to the kink width. This is yet another hint to the crucial role played by this degree of freedom in the dynamics of the system. Another important feature is the motion activation induced by noise, which was observed in the simulations and later corroborated in the CC framework.

In a wider context, we believe that the $\mathrm{CC}$ approach presented here, and the conclusions about the role of the kink width, are of a much more general applicability. This is the case, for instance, with the anomalous resonances observed in nonlinear Klein-Gordon models [42], which can only be explained by considering the width degree of freedom. Specifically within the context of rectification, we have previously shown that the existence of ratchet behavior induced by pure asymmetric temporal driving in a homogeneous system, recently demonstrated in experiments in LJJs [15], is due to the coupling between the translation and the width oscillations [14]. Based on all these experiences, we believe that the implementation of the 2CC approach can improve the results obtained in the case of spatially correlated noise $[43,44]$, where the length scale competition between the correlation length and the size of the kink takes place [43].

This formulation would also be necessary in using the CC framework for describing the transport of proteins assisted by a thermal bath provoked by ATP molecule hydrolyzation, where the width is an important quantity to take into account. On the other hand, the crucial contribution of internal degrees of freedom in the functioning of molecular motors is becoming more and more evident [32]. In this respect, our results suggest that models including this degree of freedom, in a natural way as in our case, can be proper descriptions of those phenomena. Size dependent rectification has also been reported in colloids [45] and rectification through clustering has been observed in granular gases [46], which further reinforce our conclusion that the deformation or internal degrees of freedom must be an ingredient of a good theoretical description of ratchet phenomena beyond the point particle scenario.

Finally, we want to stress that our design of a soliton system with ratchet behavior is a very simple one, which can be implemented in actual experiments and devices such as LJJs, for instance. Another field where the current state of the art allows one to apply this result is that of engineered molecular motors, demonstrated, e.g., in [47]. In this case, our proposal may apply to the design of biomolecular devices with medical applications. Generally speaking, this type of approach to rectification can be of interest for applications in which it is necessary to have a tunable rectifier tailored for a specific regime. Of course, experimental verification of our predictions is needed to ascertain the accuracy of our results. We hope that this work stimulates experiments in this direction. Indeed the research reported here opens perspectives in the design of ratchet devices for more complicated extended nonlinear systems, such as general coupled chains [48]. Of particular interest in this class are stacked LJJs [49], although there are many other systems with potential applications in different areas. Work along this line is in progress.

\section{ACKNOWLEDGMENTS}

This work has been supported by the Ministerio de Ciencia y Tecnología of Spain through Grant Nos. BFM200307749-C05-01 and FIS2004-01001 (A.S.), and by the International Research Training Group "Nonequilibrium Phenomena and Phase Transitions in Complex Systems" (DFG, Germany). The authors also want to thank N. R. Quintero for comments.

\section{APPENDIX A: COLLECTIVE COORDINATES: FIRST APPROACH}

In order to apply the GTWA first proposed in [50] we rewrite Eq. (1) adding Gaussian white noise

$$
\begin{gathered}
\dot{\phi}=\frac{\delta H}{\delta \psi}, \\
\dot{\psi}=-\frac{\delta H}{\delta \phi}-\beta \dot{\phi}-\frac{\partial \widetilde{U}}{\partial \phi} V(x)+f(t)+\eta(x, t)
\end{gathered}
$$

with

$$
\begin{gathered}
\langle\eta(x, t)\rangle=0, \\
\left\langle\eta(x, t) \eta\left(x^{\prime}, t^{\prime}\right)\right\rangle=D \delta\left(x-x^{\prime}\right) \delta\left(t-t^{\prime}\right),
\end{gathered}
$$

where $\psi=\dot{\phi}, f(t) \equiv A \sin \left(\omega t+\delta_{0}\right), D=2 \beta k_{B} T$, and $H$ is the Hamiltonian corresponding to the unperturbed form of Eq. (1) given by

$$
H=\int_{-\infty}^{+\infty} d x\left\{\frac{1}{2} \psi^{2}+\frac{1}{2} \phi_{x}^{2}+U(\phi)\right\} .
$$

As starting point we assume that the solution has the form 


$$
\phi(x, t)=\phi_{K}(x-X(t), \dot{X}),
$$

and therefore by definition of $\psi$ we have that

$$
\psi(x, t)=\psi_{K}(x-X(t), \dot{X}, \ddot{X}) .
$$

The index $K$ refers to the kink shape, but in the following we will omit it for simplicity. Following the procedure in [50], inserting $\dot{\phi}, \dot{\psi}$ into Eqs. (A1) and (A2), we get the expressions

$$
\begin{gathered}
\frac{\partial \phi}{\partial X} \dot{X}+\frac{\partial \phi}{\partial \dot{X}} \ddot{X}=\frac{\delta H}{\delta \psi} \\
\frac{\partial \psi}{\partial X} \dot{X}+\frac{\partial \psi}{\partial \dot{X}} \ddot{X}+\frac{\partial \psi}{\partial \ddot{X}}=-\frac{\delta H}{\delta \phi}-\beta\left(\frac{\partial \phi}{\partial X} \dot{X}+\frac{\partial \phi}{\partial \dot{X}} \ddot{X}\right)-\frac{\partial \tilde{U}}{\partial \phi} V(x) \\
+f(t)+\eta(x, t) .
\end{gathered}
$$

Multiplying Eq. (A7) by $\partial \psi / \partial X$ and Eq. (A8) by $\partial \phi / \partial X$, and then subtracting both expressions and integrating, we arrive at the following equation:

$$
N \ddot{X}+M \ddot{X}=-\beta C_{1} \dot{X}-\beta C_{2} \ddot{X}+F^{\mathrm{ac}}+F^{\mathrm{stat}}+F^{\mathrm{inh}}+F^{\mathrm{st}},
$$

whose values for the coefficients and forces are given by

$$
\begin{gathered}
N=\int_{-\infty}^{\infty} d x \frac{d \phi}{\partial X} \frac{\partial \psi}{\partial \ddot{X}}, \quad F^{\mathrm{ac}}=\int_{-\infty}^{\infty} d x f(t) \frac{\partial \phi}{\partial X}, \\
C_{1}=\int_{-\infty}^{\infty} d x\left(\frac{\partial \phi}{\partial X}\right)^{2}, \quad F^{\mathrm{inh}}=-\int_{-\infty}^{\infty} d x \frac{\partial \tilde{U}}{\partial \phi} V(x) \frac{\partial \phi}{\partial X}, \\
C_{2}=\int_{-\infty}^{\infty} d x \frac{\partial \phi}{\partial X} \frac{\partial \phi}{\partial \dot{X}}, \quad F^{\mathrm{st}}=\int_{-\infty}^{\infty} d x \eta(x, t) \frac{\partial \phi}{\partial X}, \\
M=\int_{-\infty}^{\infty} d x\left(\frac{\partial \psi}{\partial \dot{X}} \frac{\partial \phi}{\partial X}-\frac{\partial \phi}{\partial \dot{X}} \frac{\partial \psi}{\partial X}\right), \\
F^{\text {stat }}=-\int_{-\infty}^{+\infty} d x\left\{\frac{\delta H}{\delta \phi} \frac{\partial \phi}{\partial X}+\frac{\delta H}{\delta \psi} \frac{\partial \psi}{\partial X}\right\}=-\int_{-\infty}^{+\infty} d x \frac{\partial \mathcal{H}}{\partial X}=-\frac{\partial E}{\partial X},
\end{gathered}
$$

where $E$ represents the energy of the system, $\mathcal{H}$ is the Hamiltonian density of Eq. (A4), and $F^{\text {stat }}$ is the static force due to the external field, equal to zero for the above Hamiltonian.

Next we consider the SG potential for the system Eqs. (A1) and (A2) for which we assume as solution the ansatz

$$
\phi(x, t)=\phi^{(0)}(\gamma[x-X(t)])=4 \arctan (\exp \{\gamma[x-X(t)]\}),
$$

where $\phi^{(0)}=4 \arctan \left\{\exp \left[\left(x-X_{0}\right) / l_{0}\right]\right\}$ is the static kink solution of the SG system, centered in $X_{0}$ and of width $l_{0}$. Here $\gamma=1 / \sqrt{1-\dot{X}^{2}}$ where we have put $l_{0}=1$ for the SG case.

Considering the previous statement for the static force and taking into account $V(x)$ from Eq. (2), we obtain

$$
\begin{gathered}
N=0, \quad F^{\mathrm{ac}}=-q f(t), \\
M=\gamma^{3} M_{0}, \quad F^{\mathrm{stat}}=0, \\
C_{1}=\gamma M_{0}, \quad F^{\mathrm{inh}}=-\frac{\partial U}{\partial X}, \\
C_{2}=0,
\end{gathered}
$$

where $M_{0}=8$ is the kink mass, $q=2 \pi$ is the topological charge, and $U(X, \dot{X})$ given by

$$
U(X, \dot{X})=2 \epsilon \sum_{n} \sum_{i=1}^{3} \frac{1}{\cosh ^{2}\left[\gamma\left(X-x_{i}-n L\right)\right]}
$$

is the effective potential. In the nonrelativistic limit $\dot{X}^{2} \ll 1$, $U(X, \dot{X}) \simeq U(X)$.

A representation for the stochastic force $F^{\text {st }}$ can be obtained from the calculation of the variance. In the case of additive noise it is allowed to make the following assumption:

$$
\begin{gathered}
\left\langle\frac{\partial \phi^{(0)}(x, t)}{\partial X} \frac{\partial \phi^{(0)}\left(x^{\prime}, t^{\prime}\right)}{\partial X} \eta(x, t) \eta\left(x^{\prime}, t^{\prime}\right)\right\rangle \\
=\frac{\partial \phi^{(0)}(x, t)}{\partial X} \frac{\partial \phi^{(0)}\left(x^{\prime}, t^{\prime}\right)}{\partial X}\left\langle\eta(x, t) \eta\left(x^{\prime}, t^{\prime}\right)\right\rangle .
\end{gathered}
$$

Hence the correlation function for $F^{\text {st }}$ can be written as

$$
\begin{aligned}
& \left\langle F^{\mathrm{st}}(t) F^{\mathrm{st}}\left(t^{\prime}\right)\right\rangle \\
& \quad=\int_{-\infty}^{\infty} \int_{-\infty}^{\infty} d x d x^{\prime} \frac{\partial \phi^{(0)}(x, t)}{\partial X} \frac{\partial \phi^{(0)}\left(x^{\prime}, t^{\prime}\right)}{\partial X}\left\langle\eta(x, t) \eta\left(x^{\prime}, t^{\prime}\right)\right\rangle,
\end{aligned}
$$

for which, taking into account the expression (A2), after some algebra we get

$$
\left\langle F^{\mathrm{st}}(t) F^{\mathrm{st}}\left(t^{\prime}\right)\right\rangle=2 \beta k_{B} T \gamma M_{0} \delta\left(t-t^{\prime}\right),
$$

i.e., $F^{\text {st }}(t)$ is a white noise with kink diffusion constant

$$
D_{K}=\gamma M_{0} D .
$$

As a consequence we obtain a nonadditive noise due to the factor $\gamma(\dot{X})$, i.e., we have a problem with multiplicative noise.

Then the equation of motion (A9) can be rewritten as

$$
\gamma^{3} M_{0} \ddot{X}+\beta \gamma M_{0} \dot{X}=-q f(t)-\frac{\partial U}{\partial X}+\sqrt{D_{K}} \xi(t)
$$

with $\langle\xi(t)\rangle=0,\left\langle\xi(t) \xi\left(t^{\prime}\right)\right\rangle=\delta\left(t-t^{\prime}\right)$. Equation (A15) in the absence of inhomogeneities and noise agrees with the results presented in [51]. The other terms that appear on the righthand side in Eq. (A15) are in correspondence with those already obtained in $[23,52]$ in the presence of impurities (nonrelativistic approach) and Gaussian white noise, respectively. The procedure used here is equivalent to the so-called adiabatic approach by using modified conservation laws [25]. 


\section{APPENDIX B: COLLECTIVE COORDINATES: SECOND APPROACH}

In order to get the $\mathrm{CC}$ equations we follow a similar procedure as in the previous section but this time we propose a solution with the form

$$
\begin{gathered}
\phi(x, t)=\phi(x-X(t), l(t)), \\
\psi(x, t)=\psi(x-X(t), l(t), \dot{X}, \dot{l})
\end{gathered}
$$

with $\psi=\dot{\phi}$, which considers the kink width as a new collective variable (see, e.g., [42]).

Inserting Eqs. (B1) and (B2) in our system Eqs. (A1) and (A2) and then multiplying the first equation by $\partial \psi / \partial X$ and the second one by $\partial \phi / \partial X$; subtracting both expressions and integrating we arrive at the following equation:

$$
\begin{gathered}
\int_{-\infty}^{+\infty} d x \frac{\partial \phi}{\partial X} \frac{\partial \psi}{\partial \dot{X}} \ddot{X}+\int_{-\infty}^{+\infty} d x[\phi, \psi] \dot{l}+\int_{-\infty}^{+\infty} d x \frac{\partial \phi}{\partial X} \frac{\partial \psi}{\partial \dot{l}} \ddot{l}-F^{\text {stat }} \\
=\int_{-\infty}^{+\infty} d x F\left(x, t, \phi, \phi_{t}, \ldots\right) \frac{\partial \phi}{\partial X}
\end{gathered}
$$

with $F\left(x, t, \phi, \phi_{t}, \ldots\right)=-\beta \dot{\phi}-(\partial \tilde{U} / \partial \phi) V(x)+f(t)+\eta(x, t)$, and

$$
\begin{gathered}
{[\phi, \psi]=\frac{\partial \phi}{\partial X} \frac{\partial \psi}{\partial l}-\frac{\partial \phi}{\partial l} \frac{\partial \psi}{\partial X}} \\
F^{\text {stat }}=-\int_{-\infty}^{+\infty} d x\left\{\frac{\delta H}{\delta \phi} \frac{\partial \phi}{\partial X}+\frac{\delta H}{\delta \psi} \frac{\partial \psi}{\partial X}\right\}=-\int_{-\infty}^{+\infty} d x \frac{\partial \mathcal{H}}{\partial X}
\end{gathered}
$$

where $\mathcal{H}$ is the Hamiltonian density of Eq. (A4) for which, as was seen before, a null value for $F^{\text {stat }}$ is obtained.

Repeating the same procedure, but now with $\partial \psi / \partial l$ and $\partial \phi / \partial l$, we get the expression

$$
\begin{gathered}
\int_{-\infty}^{+\infty} d x[\psi, \phi] \dot{X}+\int_{-\infty}^{+\infty} d x \frac{\partial \phi}{\partial l} \frac{\partial \psi}{\partial \dot{X}} \ddot{X}+\int_{-\infty}^{+\infty} d x \frac{\partial \phi}{\partial l} \frac{\partial \psi}{\partial \dot{l}} \ddot{l}-K^{\mathrm{int}} \\
=\int_{-\infty}^{+\infty} d x F\left(x, t, \phi, \phi_{t}, \ldots\right) \frac{\partial \phi}{\partial l}
\end{gathered}
$$

Following Rice [53] for the particular case of the SG model

$$
\phi(x, t)=\phi^{(0)}(x-X(t), l(t))=4 \arctan \left(\exp \left[\frac{x-X(t)}{l(t)}\right]\right),
$$

Eq. (B3) becomes

$$
M_{0} l_{0} \frac{\ddot{X}}{l}+\beta M_{0} l_{0} \frac{\dot{X}}{l}-M_{0} l_{0} \frac{\dot{X} \dot{l}}{l^{2}}=F^{\mathrm{ac}}+F^{\mathrm{inh}}+F^{\mathrm{st}}
$$

with

$$
F^{\mathrm{ac}}=\int_{-\infty}^{\infty} d x f(t) \frac{\partial \phi^{(0)}}{\partial X}=-2 \pi f(t)=-q f(t)
$$

$$
\begin{gathered}
F^{\mathrm{inh}}=-\int_{-\infty}^{\infty} d x \sin \left(\phi^{(0)}\right) V(x) \frac{\partial \phi^{(0)}}{\partial X}=-\frac{\partial U}{\partial X}, \\
F^{\mathrm{st}}=\int_{-\infty}^{+\infty} d x \eta(x, t) \frac{\partial \phi^{(0)}}{\partial X},
\end{gathered}
$$

and

$$
U(X, l)=2 \epsilon \sum_{n} \sum_{i=1}^{3} \frac{1}{\cosh ^{2}\left[\left(X-x_{i}-n L\right) / l\right]} .
$$

On the other hand, Eq. (B6) is transformed into

$$
\alpha M_{0} l_{0} \frac{\ddot{l}}{l}+\beta \alpha M_{0} l_{0} \frac{\dot{l}}{l}+M_{0} l_{0} \frac{\dot{X}^{2}}{l^{2}}=K^{\mathrm{int}}(l, \dot{l}, \dot{X})+K^{\mathrm{inh}}+K^{\mathrm{st}}
$$

with

$$
\begin{gathered}
K^{\mathrm{inh}}=-\int_{-\infty}^{\infty} d x \sin \left(\phi^{(0)}\right) V(x) \frac{\partial \phi^{(0)}}{\partial l}=-\frac{\partial U}{\partial l}, \\
K^{\mathrm{st}}=\int_{-\infty}^{\infty} d x \eta(x, t) \frac{\partial \phi^{(0)}}{\partial l},
\end{gathered}
$$

$$
K^{\text {int }}(l, \dot{l}, \dot{X})=-\int_{-\infty}^{+\infty} d x \frac{\partial \mathcal{H}}{\partial l}=-\frac{\partial E}{\partial l},
$$

where $\alpha=\pi^{2} / 12, M_{0}=8, l_{0}=1$, and

$$
E=\frac{1}{2} \frac{l_{0}}{l} M_{0} \dot{X}^{2}+\frac{1}{2} \frac{l_{0}}{l} \alpha M_{0} \dot{l}^{2}+\frac{1}{2} M_{0}\left(\frac{l_{0}}{l}+\frac{l}{l_{0}}\right) .
$$

As in the previous section we use the variances of the stochastic forces in order to obtain approximate expressions for it. Taking the assumption given by the expression (A12) we find for (B11) the correlation function

$$
\begin{aligned}
& \left\langle F^{\mathrm{st}}(t) F^{\mathrm{st}}\left(t^{\prime}\right)\right\rangle=\int_{-\infty}^{\infty} \int_{-\infty}^{\infty} d x d x^{\prime} \frac{\partial \phi^{(0)}(x, t)}{\partial X} \frac{\partial \phi^{(0)}\left(x^{\prime}, t^{\prime}\right)}{\partial X} \\
& \quad \times\left\langle\eta(x, t) \eta\left(x^{\prime}, t^{\prime}\right)\right\rangle=D \delta\left(t-t^{\prime}\right) \int_{-\infty}^{\infty} d x\left(\frac{\partial \phi^{(0)}}{\partial X}\right)^{2} \\
& =D \delta\left(t-t^{\prime}\right) \frac{l_{0}}{l} M_{0} .
\end{aligned}
$$

In what follows similar expressions to Eq. (A12) valid for additive noise are used in order to calculate other correlation functions like 


$$
\begin{aligned}
& \left\langle K^{\mathrm{st}}(t) K^{\mathrm{st}}\left(t^{\prime}\right)\right\rangle=\int_{-\infty}^{\infty} \int_{-\infty}^{\infty} d x d x^{\prime} \frac{\partial \phi^{(0)}(x, t)}{\partial l} \frac{\partial \phi^{(0)}\left(x^{\prime}, t^{\prime}\right)}{\partial l} \\
& \times\left\langle\eta(x, t) \eta\left(x^{\prime}, t^{\prime}\right)\right\rangle=D \delta\left(t-t^{\prime}\right) \int_{-\infty}^{\infty} d x\left(\frac{\partial \phi^{(0)}}{\partial l}\right)^{2} \\
& =D \delta\left(t-t^{\prime}\right) \frac{l_{0}}{l} \alpha M_{0}
\end{aligned}
$$

and

$$
\begin{aligned}
& \left\langle F^{\mathrm{st}}(t) K^{\mathrm{st}}\left(t^{\prime}\right)\right\rangle=\int_{-\infty}^{\infty} \int_{-\infty}^{\infty} d x d x^{\prime} \frac{\partial \phi^{(0)}(x, t)}{\partial X} \frac{\partial \phi^{(0)}\left(x^{\prime}, t^{\prime}\right)}{\partial l} \\
& \times\left\langle\eta(x, t) \eta\left(x^{\prime}, t^{\prime}\right)\right\rangle=D \delta\left(t-t^{\prime}\right) \\
& \times \int_{-\infty}^{\infty} d x \frac{\partial \phi^{(0)}}{\partial X} \frac{\partial \phi^{(0)}}{\partial l}=0 .
\end{aligned}
$$

From the latter correlation for the stochastic forces we see that these are not cross correlated.

Finally, collecting all the previous results we can rewrite Eqs. (B8) and (B13) as follows:

$$
\begin{gathered}
M_{0} l_{0} \frac{\ddot{X}}{l}+\beta M_{0} l_{0} \frac{\dot{X}}{l}-M_{0} l_{0} \frac{\dot{X} i}{l^{2}}=-\frac{\partial U}{\partial X}-q f(t)+\sqrt{\frac{D M_{0} l_{0}}{l}} \xi_{1}(t), \\
(\mathrm{B} 21) \\
\alpha M_{0} l_{0} \frac{\ddot{l}}{l}+\beta \alpha M_{0} l_{0} \frac{i}{l}+M_{0} l_{0} \frac{\dot{X}^{2}}{l^{2}}=-\frac{\partial U}{\partial l}+K^{\mathrm{int}}(l, \dot{l}, \dot{X}) \\
+\sqrt{\frac{D \alpha M_{0} l_{0}}{l}} \xi_{2}(t)
\end{gathered}
$$

with $\left\langle\xi_{i}(t)\right\rangle=0,\left\langle\xi_{i}(t) \xi_{j}\left(t^{\prime}\right)\right\rangle=\delta_{i j} \delta\left(t-t^{\prime}\right)$, for $i, j=1,2$.

A feature of particular interest in these new equations is the presence of stochastic forces which are multiplicative white noises dependent on the kink width variable.

The method described here using the technique of projection is equivalent to the variational calculations of the momentum and the energy of the system for perturbed nonlinear Klein-Gordon systems of the form of Eqs. (A1) and (A2) and with a Hamiltonian of the form of Eq. (A4) (see [42] for details). Another procedure and derivation has been recently presented in [54].
[1] J. Maddox, Nature (London) 365, 203 (1993); 368, 287 (1994); F. Jülicher and J. Prost, Rev. Mod. Phys. 69, 1269 (1997).

[2] C. S. Lee, B. Jankó, I. Derényi, and A. L. Barabasi, Nature (London) 400, 337 (1999); J. E. Villegas, S. Savel'ev, F. Nori, E. M. Gonzalez, J. V. Anguita, R. Garcia, and J. L. Vicent, Science 302, 1188 (2003).

[3] H. Linke, W. Sheng, A. Löfgren, H. Xu, P. Omling, and P. E. Lindelof, Europhys. Lett. 44, 341 (1998); H. Linke, T. E. Humphrey, A. Löfgren, A. O. Sushkov, R. Newbury, R. P. Taylor, and P. Omling, Science 286, 2314 (1999).

[4] J. B. Majer, J. Peguiron, M. Grifoni, M. Tusveld, and J. E. Mooij, Phys. Rev. Lett. 90, 056802 (2003).

[5] R. Bartussek, P. Hänggi, and J. G. Kissner, Europhys. Lett. 28, 459 (1994).

[6] P. Hänggi and R. Bartussek, in Nonlinear Physics of Complex Systems, edited by J. Parisi, S. C. Müller, and W. Zimmermann Lecture Notes in Physics Vol. 476 (Springer, Berlin, 1996) pp. 294-308.

[7] T. E. Dialynas, K. Lindenberg, and G. P. Tsironis, Phys. Rev. E 56, 3976 (1997).

[8] P. Reimann, Phys. Rep. 361, 57 (2002).

[9] P. Reimann and P. Hänggi, Appl. Phys. A: Mater. Sci. Process. 75, 169 (2002).

[10] F. Marchesoni, Phys. Rev. Lett. 77, 2364 (1996); A. V. Savin, G. P. Tsironis, and A. V. Zolotaryuk, Phys. Lett. A 229, 279 (1997); Phys. Rev. E 56, 2457 (1997).

[11] F. Falo, P. J. Martínez, J. J. Mazo, and S. Cilla, Europhys. Lett. 45, 700 (1999).

[12] F. Falo, P. J. Martínez, J. J. Mazo, T. P. Orlando, and E. Trías, Appl. Phys. A: Mater. Sci. Process. 75, 263 (2002).

[13] S. Flach, Y. Zolotaryuk, A. E. Miroshnichenko, and M. V.
Fistul, Phys. Rev. Lett. 88, 184101 (2002); M. Salerno and Y. Zolotaryuk, Phys. Rev. E 65, 056603 (2002).

[14] L. Morales-Molina, N. R. Quintero, F. G. Mertens, and A. Sánchez, Phys. Rev. Lett. 91, 234102 (2003).

[15] A. Ustinov, C. Coqui, A. Kemp, Y. Zolotaryuk, and M. Salerno, Phys. Rev. Lett. 93, 087001 (2004).

[16] M. Salerno and N. R. Quintero, Phys. Rev. E 65, 025602 (2002); N. R. Quintero, B. Sánchez-Rey, and M. Salerno, e-print nlin.SI/0405023.

[17] G. Carapella, Phys. Rev. B 63, 054515 (2001); G. Carapella and G. Costabile, Phys. Rev. Lett. 87, 077002 (2001).

[18] L. Morales-Molina, F. G. Mertens, and A. Sánchez, Eur. Phys. J. B 37, 79 (2004).

[19] A. Sánchez, A. R. Bishop, and F. Domínguez-Adame, Phys. Rev. E 49, 4603 (1994); A. Sánchez and A. R. Bishop, SIAM Rev. 40, 579 (1998).

[20] S. Cilla and L. M. Floría, Physica D 113, 157 (1998).

[21] M. V. Satarić, J. A. Tuszyński, and R. B Źakula, Phys. Rev. E 48, 589 (1993); M. V. Satarić and J. A. Tuszyński, ibid. 67, 011901 (2003).

[22] Yu. S. Kivshar, Z. Fei, and L. Vázquez, Phys. Rev. Lett. 67, 1177 (1991); Z. Fei, Yu. S. Kivshar and L. Vázquez, Phys. Rev. A 46, 5214 (1992).

[23] S. Gredeskul, Yu. S. Kivshar, L. K. Maslov, A. Sánchez, and L. Vázquez, Phys. Rev. A 45, 8867 (1992).

[24] D. W. McLaughlin and A. C. Scott, Phys. Rev. A 18, 1652 (1978).

[25] Yu. S. Kivshar and B. A. Malomed, Rev. Mod. Phys. 61, 763 (1989).

[26] A. A. Golubov, A. V. Ustinov, and I. L. Serpuchenko, Phys. Lett. A 130, 107 (1988); A. A. Golubov, I. L. Serpuchenko and A. V. Ustinov, Sov. Phys. JETP 67, 1256 (1988). 
[27] M. Aldana-González, G. Cocho, H. Larralde and G. MartínezMekler, J. Theor. Biol. 220, 27 (2003).

[28] Yu. S. Kivshar, A. Sánchez, O. Chubykalo, A. M. Kosevich, and L. Vázquez, J. Phys. A 25, 5711 (1992).

[29] P. Jung, J. G. Kissner, and P. Hänggi, Phys. Rev. Lett. 76, 3436,(1996); J. L. Mateos, ibid. 84, 258 (2000).

[30] W. A. Strauss and L. Vázquez, J. Comput. Phys. 28, 271 (1978).

[31] H. Y. Wang and J. D. Bao, Physica A 337, 13 (2004).

[32] R. D. Vale and R. A. Milligan, Science 288, 88 (2000).

[33] D. Dan, A. M. Jayannavar, and G. I. Menon, Physica A 318, 40 (2003).

[34] F. Marchesoni, B. Y. Zhu, and F. Nori, Physica A 325, 78 (2003).

[35] M. Barbi and M. Salerno, Phys. Rev. E 62, 1988 (2000); 63, 066212 (2001).

[36] M. O. Magnasco, Phys. Rev. Lett. 71, 1477 (1993).

[37] C. R. Doering, W. Horsthemke, and J. Riordan, Phys. Rev. Lett. 72, 2984 (1994).

[38] M. M. Millonas and M. I. Dykman, Phys. Lett. A 185, 65 (1994).

[39] I. Derenyi and T. Vicsek, Phys. Rev. Lett. 75, 374 (1995).

[40] M. San Miguel and R. Toral, in Instabilities and Nonequilibrium Structures V, edited by E. Tirapegui and W. Zeller (Kluwer, Dordrecht, 1997).

[41] S. Cilla, Ph.D. thesis, Universidad de Zaragoza (2000) (in Spanish).
[42] N. R. Quintero, A. Sánchez, and F. G. Mertens, Phys. Rev. Lett. 84, 871 (2000); Phys. Rev. E 62, 5695 (2000).

[43] F. Marchesoni, Phys. Lett. A 115, 29 (1986).

[44] O. M. Braun and Yu. S. Kivshar, Phys. Rep. 306, 1 (1998).

[45] C. Marquet, A. Buguin, L. Talini, and P. Silberzan, Phys. Rev. Lett. 88, 168301 (2002).

[46] D. van der Meer, P. Reimann, K. van der Weele, and D. Lohse, Phys. Rev. Lett. 92, 184301 (2004).

[47] G. Tsiavaliaris, S. Fujita-Backer, and D. J. Manstein, Nature (London) 427, 558 (2004).

[48] O. M. Braun, Yu. S. Kivshar, and A. M. Kosevich, J. Phys. C 21, 3881 (1988).

[49] Yu. S. Kivshar and B. A. Malomed, Phys. Rev. B 37, 9325 (1988).

[50] F. G. Mertens, H.-J. Schnitzer, and A. R. Bishop, Phys. Rev. B 56, 2510 (1997).

[51] N. R. Quintero and A. Sánchez, Phys. Lett. A 247, 161 (1998).

[52] E. Joergensen, V. P. Koshelets, R. Monaco, J. Mygind, M. R. Samuelsen, and M. Salerno, Phys. Rev. Lett. 49, 1093 (1982); D. J. Bergman, E. Ben-Jacob, Y. Imry, and K. Maki, Phys. Rev. A 27, 3345 (1983).

[53] M. J. Rice and E. J. Mele, Solid State Commun. 35, 487 (1980); M. Salerno and A. C. Scott, Phys. Rev. B 26, 2474 (1982); M. J. Rice, ibid. 28, 3587 (1983).

[54] N. R. Quintero and E. Zamora-Sillero, Physica D 197, 63 (2004). 